\title{
The UAV intelligent inspection of transmission lines
}

\author{
Linxin Li \\ School of Electrical \& Electronic Engineering, North China Electric Power University, Baoding, \\ 071003 China \\ lilinxin75@gmail.com
}

Keywords: unmanned aerial vehicle (UAV); Line inspection; Intelligent rapid mission planning

\begin{abstract}
Transmission line localities in China is topography complex. Natural conditions and artificial patrol way need to spend a lot of manpower, which is inefficient. Thus transmission inspection based on unmanned aerial vehicle (UAV) has been attracting the attention of relevant researchers since produced. This article has reviewed the development of UAV power patrol process, explored its advantages, and realized the intelligent rapid mission planning through the programming.
\end{abstract}

\section{Introduction}

Long distance transmission of electric power mainly adopts high voltage and ultrahigh voltage overhead power lines. Line safe operation is the guarantee of long-distance transmission. Running state, setting defects and condition of the transmission line will affect the stable operation, so the monitoring of electric power line is very important. The traditional patrol method mainly rely on human monitoring. Artificial visual cost a lot of manpower material resources, which is inefficient. As a result of the personnel experience and personal qualities of different, monitoring effect is difficult to standardization.A new technology of moving robot, which is promising. However, the technology is difficult, high costing as well as the poor feasibility . On the contrary,the UAV monitoring by not only the related parameters of line patrol, also analyzing the acquisition of information and existing defects through remote sensing technology.The final results show that the uav patrol can get rid of the bad weather conditions, and dangerous geographical environment.It can accurate detects data. There is a huge advantage, compared with the human visual. Therefore, it is imperative to develop the uav patrol.

\section{UAV technology}

UAV is a kind of aerial vehicle,controlling by the radio remote control equipment and its programs. It is a complex integration of the system, which is consisting of electronic, aviation, electric power, reconnaissance, geographic information, image recognition, etc.UAV can be applied to many fields such as air photography, surveying and mapping, sports, military matters and scout for combating natural disasters due to its ability to operate rapidly in upper air from a long distance. And it has been taken into operation in the pilot project launched by Central China Power Grid (CCG) and China Southern Power Grid (CSG) with the development of its control techniques. Such progress enables it to be controlled by ways of telemetry data link, GPS satellite potential and geographical matching, during the process of which, stability, simplicity, reliability, highly economic performance, weather-proof feature and lasting cruising power are shown.

Compared with manual inspection methods, the technology is more scientific and efficient, which conforms to the concept of modern science and engineering.

Modern aircraft are generally has the following features and performance indicators:

1)Flight control: The flying range can reach $5 \mathrm{~km}$ under common visual remote control, while that can be $30 \mathrm{~km}$ if such ways of control as GPS autonomous navigation and geographical matching are adopted. 
2)Take-off conditions and cruising speed: UAV can also take off even when it's sprinkling as long as the wind-force has not reached strong breeze level and at least 20 meters' smooth roadway is provided.

3)Weather conditions for flying: UAV is allowed to fly in moderate rain, common one flying under strong breeze, advanced one under fresh gale.

4)Ceiling: UAV is able to fly steadily in minimum altitude zone, with common one and advanced one reaching the highest altitude of $2 \mathrm{~km}$ and $3.5 \mathrm{~km}$ respectively.

5)Carrying Capacity: the carrying capacity of common UAV is about 1 to $5 \mathrm{~kg}$, while advanced one over $10 \mathrm{~kg}$.

6)Time of endurance and distance: common UAV can fly about 30min, while advanced one can last for about 3 to 5 hours; common UAV can fly about $30 \mathrm{~km}$, advanced one 300 to $500 \mathrm{~km}$.

The process of transmission line inspection by UAV:First, attach infrared imaging related test equipment for the UAV . Then make the UAV hovering near a line to detect local fever this defect in the transmission equipment and visual defects, at the same time record the location of defects in the tower by GPS .

\section{The basic composition of UAV}

The body part. UAV requires high sensitivity of the sensor, can adjust flight when airflow mutation status. Engine should be able to keep a larger power, to overcome the air change and balance.

Communication system. Collecting and transmitting the information in the different flight states and line inspection data, UAV use wireless frequency digital radio to communicate under the condition of long distance. Related systems real-time monitor the flight status on the ground. The operators observe and analyze the condition of transmission lines and airborne equipment through the video.

The flight control system. First, it needs to have a good stabilization function, which can accurately transfer flight parameters through high sensitive attitude sensor and pressure sensor .Second, planes drift ,which equipped with differential GPS, can be strictly controlled within a certain range. Finally, it can pass the plane environment parameters to ground operators timely, so as to ensure that the aircraft stability inspection tasks.

The GPS map. GPS map specific labels the data information of substation and transmission line,which is greatly convenient for UAV for power line inspection.

\section{UAV key technology}

Autopilot. Automated driving technology is the foundation of the UAV inspection.UAV can realize patrol in ultra distance, which can not be achieved by hand control.UAV can realize autonomous flight even taking off and landing, by geographical matching automatic control, GPS autonomous navigation and control, line tower automatic tracking technology.

Image transmission link. UAV carries some devices, such as camera, image transmission equipment, etc .Image transmission equipment will send images, measured in real time, to the ground, so as to operates can analyze them. However,it's difficult to transmit the videos and images, occupying a big space,in a wireless transmission.Objectively,it also requires videos timely and images clear.Therefore it needs to use compression algorithm, which has lots of advantage,such as little distortion rate, high compression ratio, high transmission efficiency.

Target recognition. It often using line tower automatic tracking identification technology and camera shooting imaging technology,in order to make the UAV identifying the insulator string, hardware, wire and other power equipment, as well as, hovering to capture image.In order to enable UAV to recognize such electrical equipment as insulator chain, armour clamp and wire as well as capture stable images at fixed points when it is hovering, usually, automatic tracking identification technique based on overhead line structure and fast focused imaging technique will be applied. 
Such techniques can realize instantaneous exposure so that towers, railways, woods, insulators and bolts can be seen in the HD images.

Better function is taking the image of the route into the flight control algorithms, so as to make the intelligent UAV flying along the route .

Non-contact detection technology. UAV can't like the high voltage line patrol robot contiguous detect the power transmission and distribution lines, which need keep a certain distance to detect. So, non-contact detection technology is the key of the UAV patrol. Electronic optical detecting technology, laser vibration detecting technology, infrared thermal imaging technology are more widely used recently.

\section{The advantage of unmanned aircraft transmission line patrol}

In transmission and distribution line inspection, UAV is to provide a good platform for the air, it can carry all sorts of detection device, instead of artificial visual for automated checking electric line.

Its main advantages are as follows :

1)It largely overcome the terrain restrictions, and improve the efficiency of the patrol. It can not only leave no dead Angle of 360 degrees to capture the images for analyzing, but also reduce the risk of manual labor intensity and working.

2)Compared with the conventional manual patrol,UAV,carrying the infrared imager,can probe into the latent defects and weaknesses in the bottle more readily(The defects are on the insulator, hardware and bottle, which are covert.

3)Compared with the robot patrol,the same UAV can patrol $750 \mathrm{kV}, 500 \mathrm{kV}, 330 \mathrm{kV}, 220 \mathrm{kV}$, $110 \mathrm{kV}, 35 \mathrm{kV}$ voltage level transmission line,rather than doing some changes.

4)It realize fast patrol,whose efficiency can reach dozens of times of artificial patrol patrol and robot. It can obtain image data in a short time, real-time sending back and making decision as well.

5)Compared with satellite remote sensing, it largely overcome the influence of meteorological conditions, which especially is not affected by meteorological cloud cover and revisit cycle.

6)Use and maintenance cost is low. The relevant operation technology easy to master. After a one-time investment, patrol costs little at a time. When to patrol can be decided by yourself.However, hire a helicopter and other large equipment, which not only the price of is high,but also can't promise to use at any time. It may also be subject to the weather. Besides, Helicopters are also unable to fly across rivers, forests and other.

7)It can identify a variety of transmission line defects. With the development of science and technology, automatic driving and accurate positioning function allows drones can effectively identify the key parts of accidents overhead transmission lines, the tower body, conductor and insulator, shock hammer.

\section{Intelligent rapid mission planning}

Function: we can make flight plans for the aircraft, including its take-off spots, airline, landing spots and longitude, latitude and altitude of each waypoint during its flying. We can also plan such special flight mission as circuiting and hovering for the aircraft. After it is programmed, the aircraft is able to carry out the flight mission without artificial control under the instruction of task data as long as the data is written into the chip of the aircraft through wireless data transmission module. The function of storing and reading the data file of waypoint is covered in this technique, which enormously reduce the workload of formulating the flight mission. At last, a function of typing in the location of towers is included in this system: as long as the longitude and latitude of the tower is typed in the input box, the information about the waypoint can be automatically generated on the map and the monitoring site of that tower can be worked out according to intelligent algorithm, which enables the aircraft to realize the function of detection in electrical transmission line through visual pattern. 
Realization: firstly, a MO is needed to display geographical information. According to the thought of packing, inheritance, and polymorphism in high-level language, we abstract the waypoint as a category, and then abstract each of these waypoints as an object. So, adding a waypoint into the map is equal to add an instantiated object into the program. Here, for each waypoint object, its data includes longitude, latitude, altitude, command type and so on. Of course, we use generic List $<\mathrm{T}>$ to manage these waypoints for they are in order. And because the category of waypoint is <Locationwp $>$, the data of waypoint is stored in List $<$ Locationwp $>$ (remark: here, the List $<\mathrm{T}>$, rather than the DataTable, is used to process the data of waypoint, the result of which is that the processing speed is comparatively slow but the operation is very convenient because there is a set of very practical function in it, enabling it to make precise operation on some element in DS. For example, if you want to delete some waypoint, you can invoke function Remove() from the function library directly and invoke function Add() when you want to add some waypoint.)

At last, the key point is to realize the function which ensures responding monitoring waypoint to be automatically worked out when every time a tower is added. Due to the only data we can get are longitude, latitude and altitude when each time a waypoint is added, the key to working out responding multi-monitoring points around it lies in determining the strike of wire between two towers. The longitude and latitude of the earth is equal to the coordinate axis on the ground. Considering the monitoring scope is small and the distance between towers are relatively short, we can approximately view the longitude and latitude of the map as a rectangular coordinate system, longitude being $\mathrm{x}$-axis, latitude y-axis, so that every waypoint on the map is abstracted as a point with coordinate. With the coordinate, we can link this waypoint to the former one. And the segment formed by the two points is the wire between two towers. With the coordinate system, we can quantitate the "strike" of towers, and get the slope. In this way, the strike between two towers can be easily gained, and on the basis of which, we can locate responding monitoring waypoints around each tower.

\section{Conclusion}

UAVs are required very demanding because of the particularity of electric power transmission and distribution lines. Key technical problems such as target recognition and non-contact detection, autopilot, image transmission link, all need target to develop.With the advance of science and technology,accurate, reliable, convenient and efficient UAV patrol will be widely used in electric power system, in the near future.

The workload of traditional artificial methods, of which condition is very poor, is big.And it would be very difficult for us to overcome geographical conditions, weather conditions. UAV, applied to the transmission and distribution line inspection,can not only greatly improve the efficiency of inspection, but also get scientific and accurate, standardized test results. As the transmission reliability index of increasingly high demand, UAV patrol will play more important role in the field of electric power supervision. Believe in the near future, UAV patrol will be popularized and applied widely.

\section{Reference}

[1] L.Han,Z.H.Lin,M.W.Tang,Central China Electric Power,(2011)10:1657-1660

[2] M.W.Tang,L.H.Dai,Z.H.Lin,Electric Power(2013)03:35-38

[3] L.Li,X.J.Xu,B.Li,Hunan Electric Power(2012)01:44-47

[4] D.M.Yu,Y.Wu,F.D.Chen,Power System Technology(2010)02:29-32

[5] B.Q.Li,Q.Wang,B.H.Wang,Shangdong Electric Power(2010)02:29-3

[6] X.B.Chen,Y.L.Ma,Z.J.Xu,Southern Power System Technology(2008)06:59-61.

[7] J.L.Niu,S.Z.Lin,G.Q.Chen,Electronics Optics \& Control(2014)03:88-91 